\title{
Predictors of Premenstrual Syndrome among Female Students at Governmental Secondary Schools in Jeddah, Saudi Arabia: A Cross-sectional Study Iman Mohmad Wahby Salem ${ }^{1,2}$, Mashael Yahya Alsamti ${ }^{3}$, Manal Abdulaziz Murad ${ }^{4}$ \\ ${ }^{1}$ Community Medicine, Saudi Arabia-King Abdulaziz University - Rabigh, ${ }^{2}$ Community Medicine Department, Egypt- Al Azhar University, ${ }^{3}$ The Joint Program of Family Medicine, Jeddah, ${ }^{4}$ Family Medicine Department, \\ Faculty of Medicine,King Abdulaziz University, Jeddah,Saudi Arabia \\ Corresponding author: Mashael Yahya Alsamti, email:mashaelalsamti@hotmail.com
}

\section{ABSTRACT}

Background: The disorders related to menstruation are the greatest common gynecologic diseases. Premenstrual syndrome (PMS) represents a common problem among females from all age groups due to several factors. It can deteriorate their quality of life and social life. Objective: Assessment of the prevalence and predictors of premenstrual syndrome among female students at governmental secondary schools in Jeddah, Saudi Arabia, 2018.

Materials and methods: The governmental female secondary students were the population for this study which included 64926 students. The sample size is calculated using Rao soft calculator. The schools and students were chosen by a multistage probability sampling method. Slight modification and validation by three consultants to construct the questionnaire.

Results: Four hundred female students were included with mean age of $16.7 \pm 1.16$ years old. The prevalence of PMS was $37.0 \%$ (95\% CI=32.3\% - 41.9\%). There were more participants in the 3rd year (35.8\% versus $24.6 \%$, $\mathrm{p}=0.00)$ and more Saudis (55.4\% versus 44.4\%, $\mathrm{p}=0.00$ ) in the PMS group. In Saudi Arabia, other studies showed variable prevalence figures compared to those reported in our study. 35.6\% of medical students at King Faisal University had a family history of dysmenorrhea as a risk factor of PMS.

Conclusion: PMS is prevalent among one-third of secondary school students, Jeddah. Menstrual pain was the most common symptom, passive or active smoking and family history of dysmenorrhea were the predictors of PMS.

Keywords: Premenstrual syndrome, Female secondary schools students, Dysmenorrhea.

\section{INTRODUCTION}

The disorders related to menstruation are the greatest common gynecologic diseases ${ }^{(1)}$. PMS disturbs the daily lives of women and can deteriorate their quality of life and social life ${ }^{(1-3])}$. In some women, these manifestations may be excessive and become a cause of misery, absenteeism and family disharmony ${ }^{(2)}$. The severity of premenstrual syndrome symptoms is associated with its duration and how it ruins the daily lives of women ${ }^{(4)}$. Premenstrual syndrome (PMS) is characterized by a varied range of emotional $\&$ physical symptoms and behavioral changes, occurring during the luteal phase of the menstrual cycle and resolve at or within a few days after the onset of menstruation ${ }^{(1-6)}$.

The PMS was defined by the American College of Obstetricians and Gynecologists (ACOG) as "a clinical condition characterized by the cyclic presence of at least one of the physical and emotional symptoms unrelated to any organic disease that appear during the 5 days before menses in each of the three prior menstrual cycles and disappear within 4 days of the onset of menses, without recurrence until at least cycle day $13^{(5)}$. More than $90 \%$ of women of childbearing have premenstrual symptoms but a minimal proportion of female meet characters for PMS ranging from $35.6 \%$ to $96.6 \%$ worldwide ${ }^{(1-8)}$. The prevalence varied, due to cultural characteristics, sample differences and diagnostic methods. The highest rate is among the 20 to 24-year old age group then it decreases increasingly after that ${ }^{(1-8)}$. Also, PMS characterized by somatic and psychological symptoms. Somatic symptoms include abdominal bloating, headache, edema, breast tenderness, joint or muscle pain, and weight gain. While, psychological symptoms include anxiety, social withdrawal, anger, irritability, confusion, and depression. $^{(5)}$

Known risk factors for PMS are hormonal imbalance, genetic factors, lifestyle, diet, drugs, stress and psychological factors $(2,3,6,8)$.

In 2015, in India, Badkur and his colleagues ${ }^{(7)}$ conducted an observational follow-up study, to estimate the prevalence of PMS and its association with menstrual profiles among female students of colleges situated in Ujjain city, India. They found that the prevalence of PMS was $39.6 \%$ with negative history of mothers having any menstrual problems $(\mathrm{P}=0.04)$. In 2016, in Ethiopia, Abeje and Berhanu ${ }^{(3)}$ conducted a cross-sectional study, to assess the prominence of premenstrual syndrome and its associated factors between the secondary school students. They found that the prevalence of PMS was $81.3 \%$ of the participants. There were statistically significant associations between the occurrence of PMS and age AOR (CI) 1.20 $(1.01,1.44)$. Also, with early age of menarche $(<12$ years), AOR (95\% CI) 2.68 (1.32, 5.47). Another study in 2017, in Ethiopia, Tsegaye, and Getachew (2) conducted a cross-sectional study, to assess premenstrual dysphoric disorder and associated risk 
factors among female health science students in Wollo University. They found that the prevalence of premenstrual dysphoric disorder was $66.9 \%$. The level of dysmenorrhea was statistically significant with the premenstrual dysphoric disorder $(\mathrm{P}=0.008)$. In 2016, in Egypt, Ali and her colleagues ${ }^{(4)}$ conducted a crosssectional descriptive study, to explore genital and demographic predictors that might affect the seriousness of premenstrual syndrome among Students in Banha University. The study findings showed that type of college, mother's levels of education, family history of PMS and menstrual cycle intervals were statistically significant with the severity of PMS ( $\mathrm{P}=<$ $0.05,0.02,<0.05 \& 0.043$ respectively). In 2003, in $\mathrm{Al}$ Dammam, Saudi Arabia, Rasheed and Al-Sowielem ${ }^{(8)}$ conducted a study, to examine the association of PMS repeatedly with possible risk factors for the illness. The results revealed that $96.6 \%$ experienced at least one premenstrual symptom and $37.5 \%$ had a high symptom severity score. Premenstrual symptom rate was statistically significant with a maternal history of PMS $(\mathrm{P}<0.01)$, physical activity $(\mathrm{P}<0.01)$, mental stress $(\mathrm{P}<0.01)$, consumption of sweet-tasting foods $(\mathrm{P}<0.05)$, and coffee $(\mathrm{P}<0.05)$. In 2010, in Al Ahsa, Saudi Arabia, Balaha and his colleagues ${ }^{(6)}$ conducted a crosssectional study, to estimate the prevalence, severity, determinants of PMS and its impact among the female medical students. The study findings showed that the prevalence of PMS was $35.6 \%$ of cases, assessed as $45 \%$ mild, $32.6 \%$ moderate and $22.4 \%$ severe. Severe PMS was associated with more impairment of daily activities $(\mathrm{P}<0.004)$ and psychological distress symptoms $(\mathrm{P}<0.003)$. Older student age, rural residence, earlier age of menarche, regular cycles and positive family history are possible risk factors for PMS. In 2015, in Jeddah, Saudi Arabia, Karout (1) conducted a cross-sectional study, to assess the menstrual patterns and prevalence of menstrual problems among Saudi nursing students. The results detected a high distribution (prevalence) of PMS $(52.7 \%)$ and its implication on the daily productivity of $52.6 \%$. There was a significant relationship between PMS and some factors such as the academic year, early menarche and mother education level. Another study in Al Dammam, Saudi Arabia 2012, Al-Dabal (9) conducted a cross-sectional study to determine the prevalence and predictors of dysmenorrhea among university students. The results revealed that $35 \%$ of the female students had severe dysmenorrhea and $21 \%$ had moderate to severe PMS. Irregular menstruation was the most statistically significant association in the study.

\section{Rationale:}

Premenstrual syndrome represents a common problem among females from all age groups due to several factors ${ }^{(1-10)}$. PMS causes a significant psychosocial dysfunction as the symptoms usually occur with anxiety and other psychological symptoms. It is the most important reason for adolescent girls to recurrently miss classes and exams, to score a lower grade and even leave their learning. Moreover, it interferes with the nation's growth by increasing mood instability and reducing daily activity among women ${ }^{(2-}$ $8,10)$.

Objective: To assess the prevalence and predictors of premenstrual syndrome among female students at governmental secondary schools in Jeddah, Saudi Arabia, 2018.

\section{SUBJECTS AND METHODS Study design and setting}

This Cross-sectional study was conducted at governmental secondary schools, Jeddah, which is the second-largest city after Riyadh in Saudi Arabia and located in its western province.

According to the last statistical (1436-1437), the total number of governmental female secondary schools in Jeddah is about 141 schools, with approximately 64.926 students ${ }^{(11)}$. According to the Ministry of Education, Jeddah is divided into 4 Educational Centers: North, Central, South, and East. The number of governmental secondary schools in each educational center respectively is $(33,32,36 \text {, and } 40)^{(11)}$. The study population included female secondary students during the academic year 2018-2019.

\section{Inclusion criteria}

- Governmental Secondary School female students.

- Saudi and non-Saudi students.

\section{Exclusion criteria}

- Married or have been married.

- Primary amenorrhea

- Chronic illness (history of blood disorders, thyroid disease or any pelvic pathology).

- On medication (anticoagulants, oral contraceptive pill or intrauterine device).

- Abdominal operation.

\section{Sample size calculation:}

The governmental female secondary students were the population for this study. The population frame of the study is around 64926 students ${ }^{(11)}$. Based on this information, the sample size was calculated using Rao soft calculator, considering 50\% response distribution, the confidence level at $95 \%$ and allowable error of 0.05 . The sample size is accounted for as 382 students and inflated to 400 students to cover any uncompleted data. A control group included female students of non-PMS.

\section{Sampling technique:}

A multistage probability sampling method was applied. 
- In the $1^{\text {st }}$ stage, Jeddah' schools were clustered by educational centers (1437-1438) into 4 regions (North, Central, South, and East) ${ }^{(11)}$. A list of schools was obtained from the Ministry of Education and from each cluster one school was selected using simple random technique, making a total of four schools $\left(52^{\text {nd }}\right.$ secondary school, $100^{\text {th }}$ secondary school, $8^{\text {th }}$ secondary school and $86^{\text {th }}$ secondary school).

- In the $2^{\text {nd }}$ stage, by equal allocation, the total sample size was divided equally on the 4 chosen schools (100 students from each school).

- The $3^{\text {rd }}$ stage was applied by stratification of the chosen schools by grades, the required participants were selected based on the total number of students in each school and percentage that each grade represents in the school (proportional allocation).

- In the last stage, the required participants were chosen from the administration list in each school by using a systematic random sampling technique. As it is illustrated in the sampling procedure flow chart in figure (1).

\section{The tool of the study}

Slight modification and validation by three consultants were done to the adopted questionnaires ${ }^{(1,}$ ${ }^{12,13)}$ prior to its administration. The data were collected by subjecting the two-part interviewer-administered questionnaires to the participants between October 2018 and February 2019. Its two main parts covered the socio-demographic and menstrual characteristics. In this questionnaire, regular exercise is defined as $\geq 30$ minutes of physical activity on $\geq 5$ days a week ${ }^{(14)}$. Physical activity is graded as light $(<3$ Metabolic equivalent or METS), moderate (between 3 and $<6$ METS) and vigorous ( $\geq 6$ METS). Considering other characteristics, regular menstruation is defined as a cycle repeated once every 21-35 days with a duration of 3-7 days. ${ }^{(15)}$ The amount of menstrual bleeding is measured in points using a pictorial blood scoring chart system whereas the amount of bleeding is calculated from the last menses, having a score of 100 or greater is classified as menorrhagia ${ }^{(16)}$.

PMS is defined as presence of at least 1 of the somatic symptoms including abdominal bloating, headache, edema, breast tenderness, muscle pain, or weight gain and at least one of the effective symptoms including anxiety, social withdrawal, anger, irritability, confusion, or depression during the 5 days before menses for at least 3 menstrual cycles, end within 4 days after menses start, in the absence of any pharmacologic therapy, or interfere with some normal activities ${ }^{(5)}$.

The questionnaire, written in the English language, was filled out by the students in a classroom or computer lab via personal interview set up for the average time of 25 minutes. A pilot study was conducted from $10 \%$ of the total sample who were subsequently excluded from the result of the study.
Further modifications were observed before the actual data collection.

\section{Data Entry and Analysis}

The gathered data were analyzed using the SPSS software statistical program version 22. The univariate (descriptive) analysis was done for categorical data, which were presented as frequency (percentage), while continuous data were presented as mean and standard deviation. Bivariate analysis was done using Chi-square at the $\mathrm{P}<0.05$ level of significance. Lastly, multivariate analysis was employed through stepwise regression analysis to determine the predictors of premenstrual syndrome.

\section{Ethical Considerations:}

This study has ethical approval from the Research Ethics Committee of the Ministry of Education and the joint program of the family medicine Saudi board. Informed written consent was obtained from all participants with confidentiality.

\section{RESULTS}

\section{Socio-demographic and clinical characteristics}

Four hundred female students were included; mean age was $16.7 \pm 1.16$ years old, $36.8 \%$ were in $1^{\text {st }}$ year and $34.5 \%$ in $2^{\text {nd }}$ year. The majority were living with their two parents $(88.8 \%)$. Parents' educational level showed $24.8 \%$ and $36.5 \%$ of higher education among mothers and fathers, respectively; while the majority had unemployed mothers (79.5\%). Regarding economic status, $54.8 \%$ were of low socioeconomic class (monthly income $<10,000$ SAR) and $31.3 \%$ were of the middle class (Table 1). Clinical characteristics showed a mean age at menarche was $12.9 \pm 1.27$ years old. Mean BMI was $22.2 \pm 13.25 \mathrm{Kg} / \mathrm{m}^{2}$; with $14.6 \%$ being overweight or obese. Medical history showed that 52 (13.0\%) having a chronic disease, of whom 14 had asthma (26.9\%), 7 had irritable bowel syndrome $(13.5 \%)$ and 5 had diabetes (9.6\%). Lifestyle characteristics showed the prevalence of passive $(14.5 \%)$ and active (4.8\%) smoking, regular physical activity $(53.3 \%)$ and regular consumption of spicy food $(72.8 \%)$. Academically, the majority had high (60.8\%) or above average $(33.5 \%)$ grade, as per the past year grading (Table 1).

\section{Menstrual characteristics and PMS}

The menstrual interval was irregular among 59 (14.8\%) of the participants, including 46 cases of oligomenorrhea and 11 cases polymenorrhagia. Further, 29.3\% had an extended length of menstrual flow (> 7 days) and $16.0 \%$ had menorrhagia. Premenstrual somatic symptoms were present in $41.5 \%$, with breast tenderness (33.3\%) and abdominal bloating (6.3\%) being the most frequent ones. Premenstrual affective symptoms were present in $39.0 \%$, with angry bursts $(34.3 \%)$ and headache $(2.5 \%)$ being the most 
frequent ones (Table 2, Figure 1). Consequently, the prevalence of PMS was $37.0 \%$ (95\% CI=32.3\% $41.9 \%$ ). Menstrual pain was reported by $84.8 \%$, typically occurring in the first 2 days $(63.7 \%)$, of short duration ( $\leq 3$ days) in $84.7 \%$ of the cases and moderate or severe intensity in $43.4 \%$ and $37.2 \%$ cases, respectively (Table 3).

Distribution of sociodemographic, clinical and menstrual characteristics according to PMS

There were more participants in the $3^{\text {rd }}$ year $(35.8 \%$ versus $24.6 \%, \mathrm{p}=0.001)$ and more Saudis $(55.4 \%$ versus $44.4 \%, \mathrm{p}=0.002)$ in the PMS group compared to the non-PMS group. No statistically significant difference was observed between the two groups regarding the other sociodemographic factors such as parents' educational level or profession and family economic status. Regarding clinical and lifestyle factors, the PMS group had relatively lower BMI (mean $=20.9 \pm 3.5$ versus $22.9 \pm 16.4 \mathrm{Kg} / \mathrm{m}^{2}, \mathrm{p}=0.03$ ), compared to a nonPMS group, respectively. Additionally, smoking or passive smoking was more prevalent among the PMS group (27.0\% versus $14.7 \%, \mathrm{p}=0.002)$ (Table 4).

Regarding menstrual factors, the PMS group had a relatively younger age at menarche (mean $=12.7 \pm 1.28$ versus $13.0 \pm 1.26$ years old, $\mathrm{p}=0.041)$, compared to a non-PMS group, respectively. Further, PMS was associated with higher prevalence of menorrhagia $(23.6 \%$ versus $12.3 \%, p=0.00)$, dysmenorrhea $(91.2 \%$ versus $81.0 \%, \mathrm{p}=0.00$ ) and family history of dysmenorrhea $(51,4 \%$ versus $26.5 \%, p=0.00)$. Among participants with menstrual pain, those from PMS had more severe pain $(\mathrm{p}=0.02)$ and reported more frequent emotional impact, limited Activities of daily life (ADL) and school absenteeism related to the pain (Table 4).

\section{Predictors of PMS}

Multivariate regression showed passive or active smoking $(\mathrm{OR}=2.21, \mathrm{p}=0.005)$, family history of dysmenorrhea $(\mathrm{OR}=1.58, \mathrm{p}=0.050)$ and the presence of one impact of menstrual pain $(\mathrm{OR}=1.81, \mathrm{p}=0.029)$ as being independently associated with PMS (Table 5).

TABLES AND FIGURES

Table (1): Socio-demographic and clinical characteristics of the studied population $(\mathrm{N}=400)$

\begin{tabular}{|c|c|c|c|}
\hline Variable & Level & Freq. & $\%$ \\
\hline \multicolumn{4}{|l|}{ Sociodemographic data } \\
\hline Age (years) & Mean, SD & 16.7 & 1.16 \\
\hline \multirow{3}{*}{$\begin{array}{l}\text { The academic year of } \\
\text { secondary school }\end{array}$} & $1^{\text {st }}$ & 147 & 36.8 \\
\hline & $2^{\text {nd }}$ & 138 & 34.5 \\
\hline & $3^{\text {rd }}$ & 115 & 28.8 \\
\hline \multirow[t]{2}{*}{ Nationality } & Saudi & 194 & 48.5 \\
\hline & Non-Saudi & 206 & 51.5 \\
\hline \multirow[t]{2}{*}{ Area of residency in Jeddah } & Northern \& Southern & 205 & 51.3 \\
\hline & Central \& Eastern & 195 & 48.8 \\
\hline \multirow[t]{4}{*}{ Living with } & Both parents & 355 & 88.8 \\
\hline & Mother & 32 & 8.0 \\
\hline & Father & 10 & 2.5 \\
\hline & Other & 3 & 0.8 \\
\hline \multirow[t]{4}{*}{ Mother's Education } & Illiterate or Read and Write & 40 & 10.0 \\
\hline & Primary & 33 & 8.3 \\
\hline & Intermediate and Secondary & 228 & 57.0 \\
\hline & Higher education & 99 & 24.8 \\
\hline \multirow[t]{4}{*}{ Father's Education } & Illiterate or Read and Write & 19 & 4.8 \\
\hline & Primary & 25 & 6.3 \\
\hline & Intermediate and Secondary & 210 & 52.5 \\
\hline & Higher education & 146 & 36.5 \\
\hline \multirow[t]{7}{*}{ Mother's occupation } & Unemployed & 318 & 79.5 \\
\hline & Civilian Sector & 2 & 0.5 \\
\hline & Medical \& Healthcare Sector & 6 & 1.5 \\
\hline & Educational Sector & 40 & 10.0 \\
\hline & Financial Sector & 2 & 0.5 \\
\hline & Private Sector & 25 & 6.3 \\
\hline & Retired & 7 & 1.8 \\
\hline \multirow[t]{2}{*}{ Father's occupation } & Unemployed & 14 & 3.5 \\
\hline & Civilian Sector & 39 & 9.8 \\
\hline
\end{tabular}


ejhm.journals.ekb.eg

\begin{tabular}{|c|c|c|c|}
\hline Variable & Level & Freq. & $\%$ \\
\hline & Military & 26 & 6.5 \\
\hline & Medical \& Healthcare Sector & 10 & 2.5 \\
\hline & Educational Sector & 32 & 8.0 \\
\hline & Financial Sector & 22 & 5.5 \\
\hline & Private Sector & 211 & 52.8 \\
\hline & Retired & 46 & 11.5 \\
\hline \multirow{3}{*}{$\begin{array}{l}\text { Monthly family income } \\
\text { (Saudi Riyal) }\end{array}$} & $<10 \mathrm{k}$ & 219 & 54.8 \\
\hline & $10 \mathrm{k}-20 \mathrm{k}$ & 125 & 31.3 \\
\hline & $>20 \mathrm{~K}$ & 56 & 14.0 \\
\hline \multicolumn{4}{|l|}{ Clinical and lifestyle data } \\
\hline Age at menarche (years) & Mean, SD & 12.9 & 1.27 \\
\hline \multirow{7}{*}{$\begin{array}{l}\text { History of chronic disease / } \\
\text { Type of disease } \\
\S\end{array}$} & No & 348 & 87.0 \\
\hline & Yes & 52 & 13.0 \\
\hline & Diabetes mellitus & 5 & 9.6 \\
\hline & Hypertension & 1 & 1.9 \\
\hline & Asthma & 14 & 26.9 \\
\hline & Irritable Bowel Syndrome & 7 & 13.5 \\
\hline & Other & 25 & 48.1 \\
\hline \multirow{4}{*}{$\begin{array}{l}\text { History of medication / } \\
\text { Type }{ }^{\S}\end{array}$} & No & 364 & 91.0 \\
\hline & Yes & 36 & 9.0 \\
\hline & Multivitamins & 14 & 38.9 \\
\hline & Other & 22 & 61.1 \\
\hline Weight (Kg) & Mean, SD & 52.4 & 10.67 \\
\hline Height $(\mathrm{cm})$ & Mean, SD & 156.7 & 11.14 \\
\hline Body Mass Index $\left(\mathrm{Kg} / \mathrm{m}^{2}\right)$ & Mean, SD & 22.2 & 13.25 \\
\hline \multirow[t]{4}{*}{ Body Mass Index (class) } & Underweight & 98 & 24.5 \\
\hline & Normal & 244 & 61.0 \\
\hline & Overweight & 43 & 10.8 \\
\hline & Obese & 15 & 3.8 \\
\hline \multirow{7}{*}{$\begin{array}{l}\text { Smoking Status / Type of } \\
\text { smoke }^{\S}\end{array}$} & Non-smoker & 323 & 80.8 \\
\hline & Passive smoker & 58 & 14.5 \\
\hline & Active smoker & 19 & 4.8 \\
\hline & Cigarette & 3 & 15.8 \\
\hline & Shisha & 2 & 10.5 \\
\hline & Hooka & 13 & 68.4 \\
\hline & Electronic & 1 & 5.3 \\
\hline \multirow{5}{*}{$\begin{array}{l}\text { Regular physical activity / } \\
\text { Level of activity }{ }^{\S}\end{array}$} & No & 187 & 46.8 \\
\hline & Yes & 213 & 53.3 \\
\hline & Light & 42 & 19.7 \\
\hline & Moderate & 153 & 71.4 \\
\hline & Vigorous & 19 & 8.9 \\
\hline \multirow[t]{6}{*}{ Spicy food / Frequency ${ }^{\S}$} & No & 109 & 27.3 \\
\hline & Yes & 291 & 72.8 \\
\hline & 1-2 days/week & 114 & 39.0 \\
\hline & 3-5 days/week & 79 & 27.1 \\
\hline & Everyday & 70 & 24.0 \\
\hline & Other & 29 & 9.9 \\
\hline \multirow[t]{4}{*}{ Past year overall grade } & $90-100 \%$ & 243 & 60.8 \\
\hline & $75-89 \%$ & 134 & 33.5 \\
\hline & $60-74 \%$ & 21 & 5.3 \\
\hline & $0-59 \%$ & 2 & 0.5 \\
\hline
\end{tabular}

Values are frequency/percentage, expect if otherwise specified. ${ }^{\S}$ Percentages are calculated on the concerned subcategory. 
ejhm.journals.ekb.eg

Table (2): Menstrual characteristics of the studied population $(\mathrm{N}=400)$

\begin{tabular}{llcc}
\hline Variable & Level & Freq. & \% \\
Menstrual interval & Regular (21-35 days) & 341 & 85.3 \\
& Irregular (<21 or $>35$ days) & 59 & 14.8 \\
& Oligomenorrhea & 46 & 78 \\
& Polymenorrhagia & 11 & 18.6 \\
& Other & 2 & 3.4 \\
\hline Length of menstrual flow & $<3$ & 25 & 6.3 \\
(days) & $3-7$ & 258 & 64.5 \\
& $>7$ & 117 & 29.3 \\
\hline Degree of bleeding & Normal & 336 & 84.0 \\
& Heavy (Menorrhagia) & 64 & 16.0 \\
\hline Premenstrual somatic & Present (at least one) & 166 & 41.5 \\
symptoms & Absent & 234 & 58.5 \\
\hline Premenstrual affective & Present (at least one) & 156 & 39.0 \\
symptoms ${ }^{\ddagger}$ & Absent & 244 & 61.0 \\
\hline PMS & Yes & 148 & 37.0 \\
& No & 252 & 63.0 \\
\hline Family history of & Yes & 168 & 42.0 \\
dysmenorrhea & No & 232 & 58.0 \\
\hline Menstrual pain & No & 61 & 15.2 \\
(dysmenorrhea) $^{\S}$ & Yes & 339 & 84.8 \\
\hline Valus are frequ
\end{tabular}

Values are frequency/percentage, expect if otherwise specified.

Characteristics of menstrual pain are detailed in Table (3). Premenstrual somatic and affective symptoms are depicted in Figure (1).

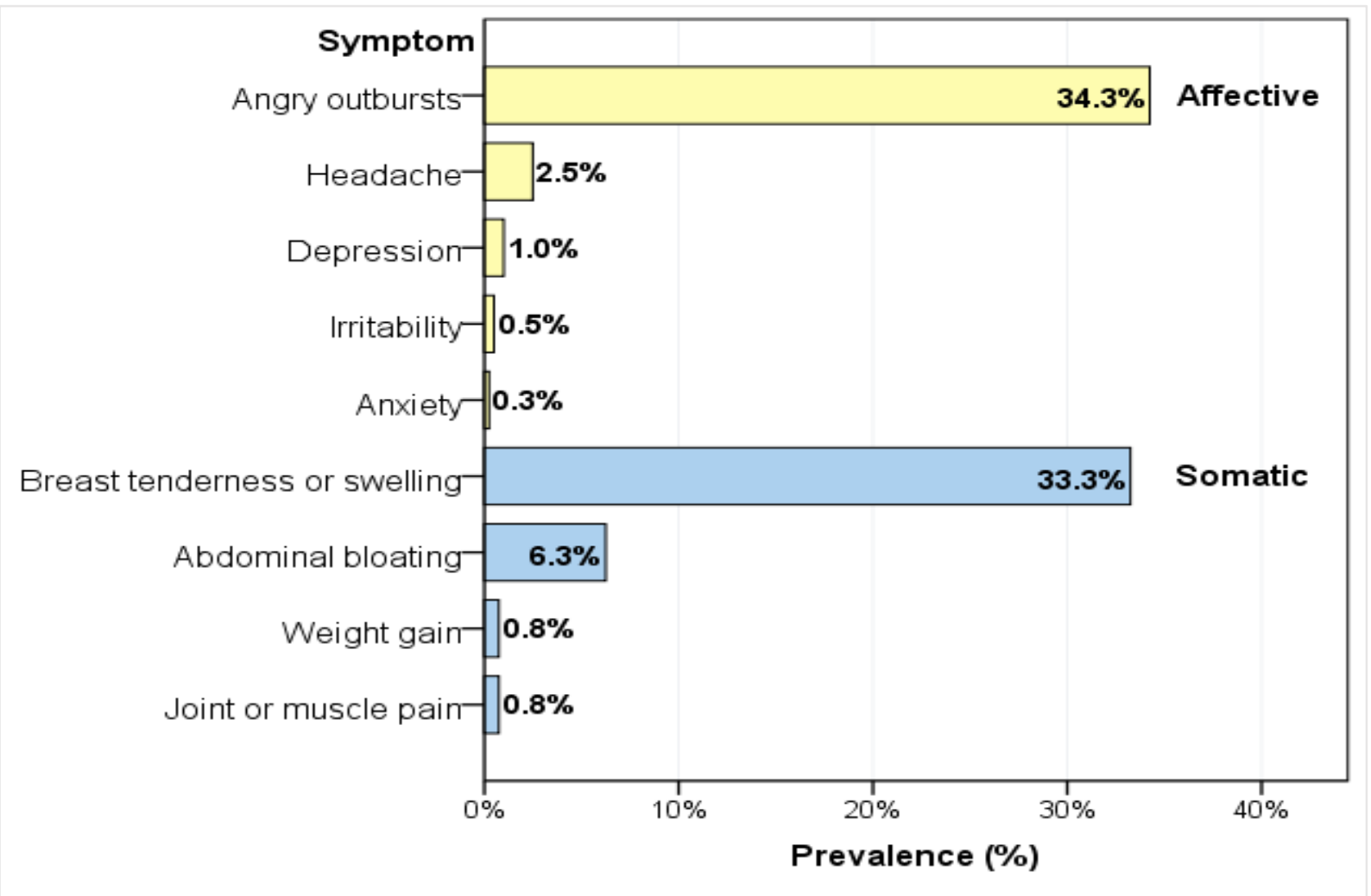

Figure (1): Prevalence of premenstrual somatic and affective symptoms. Bars represent the prevalence of the given symptom. 
Table (3): Menstrual pain characteristics ( $\mathrm{N}=339)$

\begin{tabular}{llcc}
\hline Variable & Level & Freq. & \% \\
\hline Onset during menses & Within 3 days before menses & 96 & 28.3 \\
& First 2 days of menses & 216 & 63.7 \\
& Other & 27 & 8.0 \\
\hline Duration of the pain & Normal $(\leq 3)$ & 287 & 84.7 \\
(days) & Abnormal $(>3$ days) & 52 & 15.3 \\
\hline Pattern & Continuous & 235 & 69.3 \\
& Intermittent & 104 & 30.7 \\
\hline Severity (Score) & Mild $(1-3)$ & 66 & 19.5 \\
& Moderate $(4-6)$ & 147 & 43.4 \\
& Severe $(7-10)$ & 126 & 37.2 \\
\hline
\end{tabular}

Table (4): Distribution of sociodemographic, clinical and menstrual characteristics according to Pre-Menstrual Syndrome $(\mathrm{N}=400)$

\begin{tabular}{|c|c|c|c|c|c|c|}
\hline \multirow{2}{*}{ Factor } & \multirow{3}{*}{ Level } & \multicolumn{4}{|c|}{ PMS } & \multirow{2}{*}{$\begin{array}{c}\text { p- } \\
\text { value }\end{array}$} \\
\hline & & \multicolumn{2}{|c|}{ Yes $(\mathrm{N}=148)$} & \multicolumn{2}{|c|}{ No $(\mathrm{N}=252)$} & \\
\hline Sociodemographic factors & & & & & & \\
\hline Age (years) & Mean, SD & 16.9 & 1.1 & 16.6 & 1.2 & .06 \\
\hline \multirow{3}{*}{$\begin{array}{l}\text { Academic year of } \\
\text { secondary school }\end{array}$} & $1^{\text {st }}$ & 43 & 29.1 & 104 & 41.3 & \\
\hline & $2^{\text {nd }}$ & 52 & 35.1 & 86 & 34.1 & \\
\hline & $3^{\text {rd }}$ & 53 & 35.8 & 62 & 24.6 & $.00 *$ \\
\hline \multirow[t]{2}{*}{ Nationality } & Saudi & 82 & 55.4 & 112 & 44.4 & \\
\hline & Non-Saudi & 66 & 44.6 & 140 & 55.6 & $.00 *$ \\
\hline \multirow{2}{*}{$\begin{array}{l}\text { Area of residency } \\
\text { in Jeddah }\end{array}$} & Northern \& Southern & 82 & 55.4 & 123 & 48.8 & \\
\hline & Central \& Eastern & 66 & 44.6 & 129 & 51.2 & .01 \\
\hline \multirow[t]{4}{*}{ Living with } & Both parents & $\begin{array}{c}12 \\
9\end{array}$ & 87.2 & 226 & 89.7 & \\
\hline & Mother & 14 & 9.5 & 18 & 7.1 & \\
\hline & Father & 4 & 2.7 & 6 & 2.4 & \\
\hline & Other & 1 & 0.7 & 2 & 0.8 & .86 \\
\hline \multirow{4}{*}{$\begin{array}{l}\text { Mother's } \\
\text { Education }\end{array}$} & Illiterate or Read and Write & 13 & 8.87 & 27 & 10.7 & \\
\hline & Primary & 14 & 9.5 & 19 & 7.5 & \\
\hline & Intermediate \& Secondary & 83 & 56.1 & 145 & 57.5 & \\
\hline & Higher education & 38 & 25.7 & 61 & 24.2 & .08 \\
\hline \multirow{4}{*}{$\begin{array}{l}\text { Father's } \\
\text { Education }\end{array}$} & Illiterate or Read and Write & 4 & 2.7 & 15 & 6.0 & \\
\hline & Primary & 11 & 7.4 & 14 & 5.6 & \\
\hline & Intermediate \& Secondary & 82 & 55.4 & 128 & 50.8 & \\
\hline & Higher education & 51 & 34.5 & 95 & 37.7 & .35 \\
\hline \multirow{2}{*}{$\begin{array}{l}\text { Mother's } \\
\text { occupation }\end{array}$} & Unemployed or retired & 115 & 77.7 & 210 & 83.3 & \\
\hline & Employed & 33 & 22.3 & 42 & 16.7 & .16 \\
\hline \multirow{2}{*}{$\begin{array}{l}\text { Father's } \\
\text { occupation }\end{array}$} & Unemployed or retired & 27 & 18.2 & 33 & 13.1 & \\
\hline & Employed & 121 & 81.8 & 219 & 86.9 & .67 \\
\hline \multirow{3}{*}{$\begin{array}{l}\text { Monthly family } \\
\text { income (Saudi } \\
\text { Riyal) }\end{array}$} & $<10 \mathrm{k}$ & 76 & 51.4 & 143 & 56.7 & \\
\hline & $10 \mathrm{k}-20 \mathrm{k}$ & 47 & 31.8 & 78 & 31.0 & \\
\hline & $>20 \mathrm{~K}$ & 25 & 16.9 & 31 & 12.3 & .38 \\
\hline \multicolumn{7}{|c|}{ Clinical and lifestyle factors } \\
\hline \multirow{2}{*}{$\begin{array}{l}\text { History of } \\
\text { chronic disease }\end{array}$} & No & 24 & 16.2 & 28 & 11.1 & \\
\hline & Yes & 124 & 83.8 & 224 & 88.9 & .09 \\
\hline \multirow{2}{*}{$\begin{array}{l}\text { History of } \\
\text { medication }\end{array}$} & No & 14 & 9.5 & 22 & 8.7 & \\
\hline & Yes & 134 & 90.5 & 230 & 91.3 & .47 \\
\hline Weight & Mean, SD (Kg) & 52.1 & 10.2 & 52.5 & 10.9 & .73 \\
\hline
\end{tabular}


ejhm.journals.ekb.eg

\begin{tabular}{|c|c|c|c|c|c|c|}
\hline \multirow{3}{*}{$\begin{array}{l}\text { Factor } \\
\text { Height }\end{array}$} & \multirow{3}{*}{$\begin{array}{l}\text { Level } \\
\text { Mean, SD (cm) }\end{array}$} & \multicolumn{4}{|c|}{ PMS } & \multirow{3}{*}{$\begin{array}{c}\begin{array}{c}\text { p- } \\
\text { value }\end{array} \\
.18\end{array}$} \\
\hline & & \multicolumn{2}{|c|}{ Yes $(\mathrm{N}=148)$} & \multicolumn{2}{|c|}{ No $(\mathrm{N}=252)$} & \\
\hline & & 157.6 & 6.6 & 156.1 & 13.0 & \\
\hline Body Mass Index & Mean, SD $\left(\mathrm{Kg} / \mathrm{m}^{2}\right)$ & 20.9 & 3.5 & 22.9 & 16.4 & $.03 *$ \\
\hline \multirow{2}{*}{$\begin{array}{l}\text { Smoking } \\
\text { Status / Type } \\
\text { of smoke }^{\S}\end{array}$} & $\begin{array}{l}\text { Active or passive } \\
\text { smoker }\end{array}$ & 40 & 27.0 & 37 & 14.7 & \\
\hline & Non-smoker & 108 & 73.0 & 215 & 85.3 & $.00 *$ \\
\hline \multirow{2}{*}{$\begin{array}{l}\text { Regular } \\
\text { physical } \\
\text { activity }\end{array}$} & Yes & 78 & 52.7 & 135 & 53.6 & \\
\hline & No & 70 & 47.3 & 117 & 46.4 & .47 \\
\hline \multirow[t]{2}{*}{ Spicy food } & Yes & 104 & 70.3 & 187 & 74.2 & \\
\hline & No & 44 & 29.7 & 65 & 25.8 & .23 \\
\hline \multirow{4}{*}{$\begin{array}{l}\text { Past year } \\
\text { overall grade }\end{array}$} & $90-100 \%$ & 91 & 61.5 & 152 & 60.3 & \\
\hline & $75-89 \%$ & 52 & 35.1 & 82 & 32.5 & \\
\hline & $60-74 \%$ & 5 & 3.4 & 16 & 6.3 & \\
\hline & $0-59 \%$ & 0 & 0 & 2 & 0.8 & .43 \\
\hline \multicolumn{7}{|l|}{$\begin{array}{l}\text { Menstrual } \\
\text { factors }\end{array}$} \\
\hline Age at menarche & Mean, SD (years) & 12.7 & 1.28 & 13.0 & 1.26 & $.041 *$ \\
\hline \multirow[t]{2}{*}{ Age of Menarche } & $\leq 12$ years & 64 & 43.2 & 81 & 32.1 & \\
\hline & $>12$ years & 84 & 56.8 & 171 & 67.9 & $.001 *$ \\
\hline \multirow{2}{*}{$\begin{array}{l}\text { Menstrual } \\
\text { regularity }\end{array}$} & Regular (21-35 days) & 123 & 83.1 & 218 & 86.5 & \\
\hline & $\begin{array}{l}\text { Irregular }(<21 \text { or }>35 \\
\text { days })\end{array}$ & 25 & 16.9 & 34 & 13.5 & .21 \\
\hline \multirow{2}{*}{$\begin{array}{l}\text { Type of } \\
\text { irregularity }\end{array}$} & Oligomenorrhea & 19 & 76.0 & 28 & 82.4 & \\
\hline & Polymenorrhagia & 6 & 24.0 & 6 & 17.6 & .39 \\
\hline \multirow{2}{*}{$\begin{array}{l}\text { Length of } \\
\text { menstrual } \\
\text { flow }\end{array}$} & $\begin{array}{l}\text { Normal } \\
\text { (3-7 days) }\end{array}$ & 95 & 64.2 & 163 & 64.7 & \\
\hline & $\begin{array}{l}\text { Abnormal } \\
(<3 \text { or }>7 \text { days })\end{array}$ & 53 & 35.8 & 89 & 35.3 & .50 \\
\hline \multirow{2}{*}{$\begin{array}{l}\text { Degree of } \\
\text { bleeding }\end{array}$} & Normal & 113 & 76.4 & 221 & 87.7 & \\
\hline & Heavy (Menorrhagia) & 35 & 23.6 & 31 & 12.3 & $.00 *$ \\
\hline \multirow{2}{*}{$\begin{array}{l}\text { Family history of } \\
\text { dysmenorrhea }\end{array}$} & Yes & 76 & 51.4 & 92 & 36.5 & \\
\hline & No & 72 & 48.6 & 160 & 63.5 & $.00 *$ \\
\hline \multirow[t]{2}{*}{ Menstrual pain } & Yes & 135 & 91.2 & 204 & 81.0 & \\
\hline & No & 13 & 8.8 & 48 & 19.0 & $.00 *$ \\
\hline \multirow{3}{*}{$\begin{array}{l}\text { Pain severity } \\
\text { (Score) })^{¥}\end{array}$} & Mild (1-3) & 20 & 14.8 & 46 & 22.5 & \\
\hline & Moderate (4-6) & 56 & 41.5 & 91 & 44.6 & \\
\hline & Severe $(7-10)$ & 59 & 43.7 & 67 & 32.8 & $.02 *$ \\
\hline \multirow{6}{*}{$\begin{array}{l}\text { Impact of } \\
\text { menstrual pain } \\
¥\end{array}$} & $\begin{array}{l}\text { Emotional instability or } \\
\text { sleep disturbance }\end{array}$ & 14 & 10.4 & 13 & 6.4 & \\
\hline & Decreased social activity & 3 & 2.2 & 4 & 2.0 & \\
\hline & $\begin{array}{l}\text { Limited ADL or } \\
\text { assignments }\end{array}$ & 18 & 13.3 & 16 & 7.8 & \\
\hline & Absenteeism & 6 & 4.4 & 4 & 2.0 & \\
\hline & Multi-effects & 90 & 66.7 & 144 & 70.6 & \\
\hline & No impact & 4 & 3.0 & 23 & 11.3 & $.02 *$ \\
\hline
\end{tabular}

Values are frequency/percentage, expect if otherwise specified. ${ }^{\S}$ Percentages are calculated on the total number of patients with menstrual irregularity $(\mathrm{N}=59) .{ }^{¥}$ Analysis carried out among participants who had menstrual pain $(\mathrm{N}=339)$. ADL: Activities of daily life; $*$ statistically significant results $(\mathrm{p}<0.05)$. 
ejhm.journals.ekb.eg

Table (5): Socio-demographic and menstrual predictors of PMS (Multivariable logistic regression).

\begin{tabular}{|c|c|c|c|c|c|}
\hline \multirow{2}{*}{ Predictor / Level } & \multirow{2}{*}{ B } & \multirow{2}{*}{ Sig. } & \multirow{2}{*}{$\mathbf{O R}$} & \multicolumn{2}{|c|}{$95 \% \mathrm{CI}$} \\
\hline & & & & Inf. & Sup. \\
\hline Smoking (yes) & 0.79 & $.005^{*}$ & 2.21 & 1.27 & 3.86 \\
\hline $\begin{array}{l}\text { Family History of Dysmenorrhea } \\
\text { (yes) }\end{array}$ & 0.46 & $.050^{*}$ & 1.58 & 1.01 & 2.48 \\
\hline $\begin{array}{l}\text { Impact of menstrual pain } \\
\text { No Impact / no pain } \\
\text { One aspect }\end{array}$ & $\begin{array}{r}\text { Ref } \\
0.59\end{array}$ & $\begin{array}{l}.006 * \\
.029 *\end{array}$ & $\overline{-}$ & $\overline{-}$ & 3.08 \\
\hline Multiple aspects & $\begin{array}{c}- \\
1.15\end{array}$ & $.042 *$ & 0.32 & .011 & 0.96 \\
\hline Mother's occupation (Unemployed) & -0.48 & .107 & 0.62 & 0.35 & 1.11 \\
\hline
\end{tabular}

B: Adjusted regression coefficient; OR: odds ratio; CI: confidence interval; ref: a category used as a reference to calculate the OR.

\section{DISCUSSION}

Multiple physical, emotional, and behavioral changes can take place during PMS. These symptoms occur at a higher rate in young women, and they commonly manifested between puberty and the third decade of life ${ }^{(4,5)}$. The uncertainties regarding definitive PMS etiologies underscore the importance of studying PMS prevalence and its associated risk factors in different regions worldwide. The present cross-sectional study was conducted among secondary school students in Jeddah, showing a PMS prevalence of $37 \%$, where somatic and effective symptoms were prevalent among $41.5 \%$ and $39 \%$ of participants, respectively. Several sociodemographic and clinical factors were significantly associated with PMS, such as low BMI, Saudi nationality, and young age at menarche. Interestingly, active or passive smoking, as well as a family history of dysmenorrhea, predicted PMS. Besides, menstrual pain was significant among $84.8 \%$ of students and its effects, such as limited ADL and absenteeism predicted the onset of PMS.

Prevalence figures reported in the present study were relatively lower than other global estimates. In a meta-analysis of 17 prevalence-based studies, Direkvand-Moghadam et al. ${ }^{(17)}$ showed that the pooled prevalence of PMS was $47.8 \%$. The highest and lowest prevalence rates were reported in Iran (98\%) and France (12\%), respectively. Of note, the continent-based analysis revealed a significant increase in PMS prevalence in Asia than in Europe ${ }^{(17)}$. In Africa, the prevalence ranged between $66.9 \%$ and $81.3 \%$ in Ethiopia ${ }^{(2,3)}$. However, an early review of cross-sectional studies showed low prevalence estimates in nonwestern cultures (23-34\%) as compared to Western communities (71$73 \%)^{(18)}$. The demonstrated variations in population characteristics emphasize the role of the individual or community-based variations, including age, nutrition, and physical activities, in the prevalence of PMS ${ }^{(17,19)}$.

In Saudi Arabia, other studies showed variable prevalence figures compared to those reported in our study. For example, $35.6 \%$ of 250 medical students at King Faisal University, Al Ahsa had PMS as revealed using structured interviews ${ }^{(6)}$, which is concordant with our findings. However, Rasheed and Al-Sowielem (8) found significantly different outcomes among collegeaged women. The authors demonstrated a PMS prevalence of $96.6 \%$. Yet the authors indicated that these results based on the presence of at least one PMS symptom during the six months that had preceded the cross-sectional investigation. In another regional study performed in four regions in Saudi Arabia, Algahtani and Jahrami ${ }^{(20)}$ showed that almost all women (99\%) had PMS where moderate and severe symptoms were apparent in $44.6 \%$ and $5.6 \%$ of participants, respectively. The authors of such a study developed a specialized scale (the Arabic Premenstrual Syndrome Scale [APMSS] to be utilized for screening purposes among Arabic women based on the criteria of the Diagnostic and Statistical Manual of Mental Disorders, fourth edition (DSM-IV) ${ }^{(20)}$. The diagnostic criteria play also a significant role in determining the epidemiological patterns. For example, Hashim et al. ${ }^{(21)}$ found a 95\% prevalence of PMS among college students (aged 18-24 years), while the figure reduced to $35.3 \%$ after the application of DSM-IV criteria. Therefore, it can be presumed that methodological variations might have contributed to the high figures observed in some local studies. These include the methods of data collection, the study population, the used diagnostic criteria, and sampling technique. Additionally, some patient-related factors may hinder self-reporting in some areas. In essence, cultural-based variations may inhibit women to express their own symptoms. Menstruation and menstrual problems are viewed as a women's issue and would never be discussed in the presence of men, even first-degree relatives. Moreover, the notion of "diseases" or "syndromes" are frequently neglected among Arab women ${ }^{(22)}$. This might explain the higher prevalence of the condition in cross-sectional studies, where data were collected confidentially and voluntarily, rather than 
prevalence rates reported in prospective investigations ${ }^{(8)}$. As such, the impact of the selection bias should be considered in such instances.

The increased prevalence in cross-sectional studies employing a convenient sampling approach might have partly been overcome in our study by utilizing a stratified randomized sampling of secondary schools. Nonetheless, voluntary participation remained a significant limitation of our analysis. Therefore, future regional or nation-wide studies should be implemented to address the true prevalence of PMS, considering a well-designed, randomized, prospective approach. The variation in the frequencies of physical symptoms could be explained by differences in lifestyle factors and the coping practices among surveyed women. In our study, active and passive smoking predicted PMS. In the United Arab Emirates, Hashim et al. (21) demonstrated an increased risk of reporting PMS and higher severity indices among active smokers as compared to non-smokers. Besides, younger women who started smoking before 15 years of age had higher relative risks of PMS as compared to their counterparts ${ }^{(23)}$. Hormonal factors may have been implicated, such that smoking may dysregulate the levels of progesterone, estrogen, and gonadotropin (23). However, these postulations remain unconfirmed and require further validation.

A family history of dysmenorrhea was another risk factor of PMS. Based on a literature review, only Badkur et al. ${ }^{(7)}$ revealed that PMS was associated with a family history of menstrual problems in mothers and sisters. These problems included dysmenorrhea. However, other studies did not reveal such a finding. Instead, severe dysmenorrhea among female participants was found to be associated with severe premenstrual symptoms in multiple studies ${ }^{(4,24)}$. The association between familial dysmenorrhea and PMS could be explained by some behavioral changes of daughters who have been potentially learned from the mothers' symptoms ${ }^{(25)}$.

Intriguingly, the pain was the most common symptom among the respondents in the present study (84.8\%). This is following the outcomes of the vast majority of other studies in Europe and Asia ${ }^{(26-31)}$. Other types of bodily pain varied across studies. A recent study from the United Arab Emirates (21) showed that muscle/back/joint pain was the most frequent symptom. Besides, physical symptoms and effective symptoms have been the most common symptoms in other studies ${ }^{(32)}$. Coping with stress was a significant factor, where women of low socioeconomic statuses may be unable to cope with stress concomitant with their challenging environment and subsequently, their physical and psychological health may be negatively influenced ${ }^{(33)}$.

Indeed, the aforementioned findings necessitate

taking urgent actions to circumvent this problem. Basically, the implications of reproductive health should be integrated into specific educational programs targeting school and college students. This would provide accurate information to young females and support their menstrual issues and reproductive health in general. Hopefully, the recent rising trend of female empowerment and positive attitudes regarding the contribution of Saudi females in the daily life, which have been associated with rapid modernization and development of the local community might all lead to increase the levels of awareness and perception of females about the implications of PMS to initiate consultation and receive adequate treatment.

The present study had some limitations. We did not use specialized and reliable tools to assess the potential concurrent psychiatric or physical illnesses that might have affected the outcomes. Besides, data collection might have been subject to recall bias, as respondents might have memorized their recent experiences of pain rather than recalling long-term symptoms.

\section{CONCLUSION}

PMS is prevalent among one-third of secondary school students in Jeddah and has significant health problems with several implications in daily life. Menstrual pain was the most common symptom, while physical and effective symptoms were relatively equally distributed. The distribution of these symptoms showed significant variations across different studies conducted locally, regionally, and globally. Sociodemographic and cultural factors can significantly impact the prevalence and clinical patterns of PMS and this may partly explain the diversity of PMS symptomatology. In the Saudi context, it is necessary to encourage PMS selfidentification, particularly among those who are reluctant to report disease symptoms due to cultural constraints. Besides, it is important to address the burden of the disease through regular screening of its risk factors and investigating its impact on the individual's quality of life and society.

\section{REFERENCES}

1. El Karout N (2015): Prevalence and pattern of menstrual problems and relationship with some factors among Saudi nursing students. J Nurs Educ Pract., 5 (12):1-8.

2. Tsegaye D, Getachew Y (2017): Premenstrual dysphoric disorder and associated factors among female health science students in Wollo University, Ethiopia, Matern Health Neonatol Perinatol., 5:8.

3. Abeje A, Berhanu $Z$ (2019): Premenstrual syndrome and factors associated with it among secondary and preparatory school students in Debremarkos town, North-west Ethiopia, BMC Res Notes, 12: 535.

4. Ali F, Fawaz M, Elsharkawy N (2016): Reproductive and demographic Predictors' of premenstrual syndrome severity among university students. IOSR Journal of Nursing and Health Scienc, 05: 10-19. 
5. Hofmeister S, Bodden S (2016): Premenstrual Syndrome and Premenstrual Dysphoric Disorder. Am Fam Physician, 94: 23640.

6. Balaha MH, Amr MAEM, Saleh Al Moghannum M, Saab Al Muhaidab N (2010): The phenomenology of premenstrual syndrome in female medical students: a cross sectional study. Pan Afr Med J., 5: 4.

7. Badkur D, Singh S, Chauhan D, Sinha A (2018): Premenstrual syndrome and its association with menstrual profile among female students of colleges in Ujjain city, Madhya Pradesh, India. International Journal of Research in Medical Sciences , 6: 2726.

8. Rasheed P, Al-Sowielem LS (2003): Prevalence and predictors of premenstrual syndrome among college-aged women in Saudi Arabia. Ann Saudi Med., 23:381-7.

9. Al-Dabal BK, Koura MR, Al-Sowielem LS, Barayan SS (2014): Dysmenorrhea and associated risk factors among university students in Eastern Province of Saudi Arabia. World Fam Med J., 99:1-11.

10. Buddhabunyakan N, Kaewrudee S, Chongsomchai C, Soontrapa S, Somboonporn W, Sothornwit J (2017): Premenstrual syndrome (PMS) among high school students. Int $\mathbf{J}$ Womens Health, 9:501-5.

11. El Tawila, Mahmoud M (2017): Dietary exposure to acrylamide from cafeteria foods in Jeddah schools and associated risk assessment. Journal of the Science of Food and Agriculture ,97(13): 4494-4500.

12. Gebeyehu MB, Mekuria AB, Tefera YG et al. (2017): Prevalence, impact, and management practice of dysmenorrhea among University of Gondar Students, Northwestern Ethiopia: a cross-sectional study." International journal of reproductive medicine https://www.hindawi.com/journals/ijrmed/2017/3208276/

13. Ibrahim NK, AlGhamdi MS, Al-Shaibani AN, AlAmri FA, Alharbi HA, Al-Jadani AK et al. (2015): Dysmenorrhea among female medical students in King Abdulaziz University: Prevalence, Predictors and outcome. Pak J Med Sci., 31: 1312-7.

14. Al-Shehri FS, Moqbel MM, Al-Khaldi YM et al. (21016): Prevention and management of obesity: Saudi guideline update. Saudi J Obesity, 4: 25.

15. Albers JR, Hull SK, Wesley RM (2004): Abnormal uterine bleeding. Am Fam Physician, 69: 1915-26.

16. Magnay JL, O'Brien S, Gerlinger C, Seitz C (2018): A systematic review of methods to measure menstrual blood loss. BMC Womens Health, 18: 142.

17. Direkvand-Moghadam A, Sayehmiri K, Delpisheh A, Sattar K (2018): Epidemiology of Premenstrual Syndrome (PMS)-A Systematic Review and Meta-Analysis Study. J Clin Diagn Res., 8: $106-9$

18. A cross-cultural study of menstruation (1981): implications for contraceptive development and use. World Health Organization Task Force on Psychosocial Research in Family Planning, Special Programme of Research, Development and Research, Training in Human Reproduction. Stud Fam Plann., 12: 3-16.
19. Vishnupriya $R$, Rajarajeswaram $P$ (2011): Effects of aerobic exercise at different intensities in pre menstrual syndrome. $\mathbf{J}$ Obstet Gynaecol India, 61: 675-82.

20. Algahtani H, Jahrami H (2014): The Experience and Severity of Premenstrual Syndrome among a Saudi Sample using a newly Developed Arabic Language Scale. Arab J Psychiatr., 25: 33-9.

21. Hashim MS, Obaideen AA, Jahrami HA et al. (2019): Premenstrual Syndrome Is Associated with Dietary and Lifestyle Behaviors among University Students: A CrossSectional Study from Sharjah, UAE. Nutrients, 11: 1939.

22. Allihabi A(2019): Premenstrual Syndrome- Prevalence, Severity and Effect on Academic Performance: A Comparative Study Between Students of Medicine and Literature. J Womens Health Gyn., 6: 1-18.

23. Bertone-Johnson ER, Hankinson SE, Johnson SR, Manson JE (2008): Cigarette smoking and the development of premenstrual syndrome. Am J Epidemiol., 168: 938-45.

24. Steiner M, Peer M, Palova E, Freeman EW, Macdougall M, Soares CN (2011): The Premenstrual Symptoms Screening Tool revised for adolescents (PSST-A): prevalence of severe PMS and premenstrual dysphoric disorder in adolescents. Arch Womens Ment Health., 14: 77-81.

25. Dorn LD, Negriff S, Huang B et al. (2009): Menstrual symptoms in adolescent girls: association with smoking, depressive symptoms, and anxiety. J Adolesc Health, 44: 23743.

26. Omar K, Mohsin SS, Muthupalaniappen L, Idris IB, Amin RM, Shamsudin K (2009): Premenstrual symptoms and remedies practiced by Malaysian women attending a rural primary care clinic. Afr J Prim Health Care Fam Med., 1: 018.

27. Pal SA, Dennerstein L, Lehert $P$ (2011): Premenstrual symptoms in Pakistani women and their effect on activities of daily life. J Pak Med Assoc., 61: 763-8.

28. Gamal AM, Shahin MA (2015): Premenstrual Syndrome, Associated Symptoms and Evidence-Based Nursing Management: A Comparative Study Between Rural Menoufia Governorate (Egypt) and Hodidha Governorate (Yemen). Am J Nurs Sci., 4: 84-93.

29. Obeidat BA, Alchalabi HA, Abdul-Razzak KK, Al-Farras MI (2012): Premenstrual symptoms in dysmenorrheic college students: prevalence and relation to vitamin D and parathyroid hormone levels. Int J Environ Res Public Health, 9: 4210-22.

30. Thu M, Diaz E, Kapaw S (2006): Premenstrual syndrome among female university students in Thailand. AU JT., 9: 15862.

31. Goker A, Artunc-Ulkumen B, Aktenk F, Ikiz N (2015): Premenstrual syndrome in Turkish medical students and their quality of life. J Obstet Gynaecol., 35: 275-8.

32. Derman O, Kanbur NO, Tokur TE, Kutluk T (2004): Premenstrual syndrome and associated symptoms in adolescent girls. Eur J Obstet Gynecol Reprod Biol., 116: 201-6.

33. Grant KE, Compas BE, Thurm AE, McMahon SD, Gipson PY, Campbell AJ et al. (2006): Stressors and child and adolescent psychopathology: evidence of moderating and mediating effects. Clin Psychol Rev., 26: 257-83. 